\title{
Ground-state densities from the Rayleigh-Ritz variation principle and from density-functional theory
}

\author{
Simen Kvaa* and Trygve Helgakent \\ Centre for Theoretical and Computational Chemistry, \\ Department of Chemistry, University of Oslo, \\ P.O. Box 1033 Blindern, N-0315 Oslo, Norway
}

(Dated: May 24, 2022)

The relationship between the densities of ground-state wave functions (i.e., the minimizers of the Rayleigh-Ritz variation principle) and the ground-state densities in density-functional theory (i.e., the minimizers of the Hohenberg-Kohn variation principle) is studied within the framework of convex conjugation, in a generic setting covering molecular systems, solid-state systems, and more. Having introduced admissible density functionals as functionals that produce the exact ground ground-state energy for a given external potential by minimizing over densities in the HohenbergKohn variation principle, necessary sufficient conditions on such functionals are established to ensure that the Rayleigh-Ritz ground-state densities and the Hohenberg-Kohn ground-state densities are identical. We apply the results to molecular systems in the Born-Oppenheimer approximation. For any given potential $v \in L^{3 / 2}\left(\mathbb{R}^{3}\right)+L^{\infty}\left(\mathbb{R}^{3}\right)$, we establish a one-to-one correspondence between the mixed ground-state densities of the Rayleigh-Ritz variation principle and the mixed ground-state densities of the Hohenberg-Kohn variation principle when the Lieb density-matrix constrainedsearch universal density functional is taken as the admissible functional. A similar one-to-one correspondence is established between the pure ground-state densities of the Rayleigh-Ritz variation principle and the pure ground-state densities obtained using the Hohenberg-Kohn variation principle with the Levy-Lieb pure-state constrained-search functional. In other words, all physical groundstate densities (pure or mixed) are recovered with these functionals and no false densities (i.e., minimizing densities that are not physical) exist. The importance of topology (i.e., choice of Banach space of densities and potentials) is emphasized and illustrated. The relevance of these results for current-density-functional theory is examined.

\footnotetext{
* simen.kvaal@kjemi.uio.no
}

$\dagger$ t.u.helgaker@kjemi.uio.no 


\section{INTRODUCTION}

The concept of modeling an electron gas and more generally all electronic systems using only the density $\rho$ goes back to the early days of quantum mechanics and the independent work of Tomas and Fermi in 1927 [1, 2]. However, it was only with the seminal work of Hohenberg and Kohn in 1964 that density-functional theory (DFT) was shown to be - in principle, at least - an exact theory [3]. In 1983, Lieb formulated DFT rigorously for electronic systems in $\mathbb{R}^{3}$, using concepts from convex analysis [4]. Today, DFT is the most popular computational method for many-electron systems in chemistry and solid-state physics.

In an external electrostatic scalar potential $v$, the ground-state energy $E(v)$ of an $N$-electron system is obtained from the Rayleigh-Ritz variation principle

$$
E(v):=\inf _{\psi}\langle\psi|\hat{H}(v)| \psi\rangle
$$

where $\hat{H}(v)$ is the Hamiltonian and the infimum extends over all $L^{2}$-normalized wave functions for which the expectation value makes sense. With each minimizing ground-state wave function $\psi$ in Eq. (11), there is an associated ground-state density $\rho$. In DFT, the ground-state energy $E(v)$ is instead obtained from the Hohenberg-Kohn variation principle,

$$
E(v)=\inf _{\rho}\left(F_{0}(\rho)+(v \mid \rho)\right)
$$

where $F_{0}(\rho)$ is the universal functional and the minimization is over all densities for which the interaction $(v \mid \rho)=\int v(\mathbf{r}) \rho(\mathbf{r}) \mathrm{d} \mathbf{r}$ is meaningful. Note that we have not specified the spatial domain nor the nature of the external potential $v$. Thus, the minimization problems (1) 2) are so far not fully defined from a mathematical perspective. Moreover, the functional $F_{0}$ is not unique - any functional $F_{0}$ that gives the correct groundstate energy in the Hohenberg-Kohn variation principle is said to be 'admissible'. Common expositions of DFT use the Levy-Lieb constrained-search functional $F_{\mathrm{LL}}$ [4, 5], the density-matrix constrained-search functional $F_{\mathrm{DM}}[4$, and the Lieb functional $F$, all giving the same ground-state energy $E(v)$ for a large class of potentials. Specializing to electrons in $\mathbb{R}^{3}$ and assuming that the external potential $v$ is sufficiently well-behaved, these functionals are given by the following expressions:

$$
\begin{aligned}
F_{\mathrm{LL}}(\rho) & :=\inf \left\{\left\langle\psi\left|\hat{H}_{0}\right| \psi\right\rangle \mid \psi \in H_{\mathrm{S}}^{1}\left(\mathbb{R}^{3 N}\right),\|\psi\|_{2}=1, \psi \mapsto \rho\right\} \\
F_{\mathrm{DM}}(\rho) & \left.:=\inf _{\{}\left\{\operatorname{Tr}\left(\hat{H}_{0} \Gamma\right)\left|\Gamma=\sum_{k} \lambda_{k}\right| \psi_{k}\right\rangle\left\langle\psi_{k}\right|, \sum_{k} \lambda_{k}=1, \lambda_{k} \geq 0, \psi_{k} \in H_{\mathrm{S}}^{1}\left(\mathbb{R}^{3 N}\right),\left\|\psi_{k}\right\|_{2}=1, \psi \mapsto \rho\right\} \\
F(\rho) & :=\sup _{v \in X^{\prime}}(E(v)-(v \mid \rho)) .
\end{aligned}
$$

Here $H_{\mathrm{S}}^{1}\left(\mathbb{R}^{3 N}\right)$ is the first-order Sobolev space $H_{\mathrm{S}}^{1}\left(\mathbb{R}^{3 N}\right)$ with proper permutation symmetry due to spin, $\hat{H}_{0}=\hat{T}+\hat{W}$ is the sum of the kinetic and inter-electron interaction operators, and $\psi \mapsto \rho$ and $\Gamma \mapsto \rho$ indicate that the wave function $\psi$ and density matrix $\Gamma$, respectively, have density $\rho$. In the definition of the Lieb functional, $X$ is a Banach space in which densities are embedded, with $X^{\prime}$ as its dual, consisting of potentials. We note that the Lieb functional is by construction lower semi-continuous and convex, being the conjugate function to $E$ in the sense of convex analysis.

Whereas the density functionals $F_{\mathrm{LL}}, F_{\mathrm{DM}}$ and $F$ are all admissible and therefore give the same total energy in the Hohenberg-Kohn variation principle, they may have different minimizing densities. In this article, we study the relationship between the densities in the Hohenberg-Kohn and Rayleigh-Ritz variation principles. In particular, we establish what conditions must be imposed on an admissible density functional to ensure that the ground-state densities (i.e., the minimizing densities) in the Hohenberg-Kohn variation principle are the same as the densities of the ground-state wave functions (i.e., the minimizing wave functions) in the Rayleigh-Ritz variation principle.

While our treatment covers the standard DFT setting outlined above, it is motivated by a study of current-density-functional theory (CDFT), where the $N$-electron system is subject to an external magnetic vector potential $\mathbf{A}$ in addition to the scalar potential $v$ [6 8], introducing the paramagnetic current density $\mathbf{j}_{\mathrm{p}} \in \mathbf{L}^{1}\left(\mathbb{R}^{3}\right)$ as an additional variable in the Hohenberg-Kohn variation principle:

$$
E(v, \mathbf{A})=\inf _{\rho, \mathbf{j}_{\mathrm{p}}}\left(F_{0}\left(\rho, \mathbf{j}_{\mathrm{p}}\right)+\left(v+\frac{1}{2} A^{2} \mid \rho\right)+\left(\mathbf{A} \mid \mathbf{j}_{\mathrm{p}}\right)\right)
$$


where $\left(\mathbf{A} \mid \mathbf{j}_{\mathrm{p}}\right)=\int \mathbf{A}(\mathbf{r}) \cdot \mathbf{j}_{\mathrm{p}}(\mathbf{r}) \mathrm{d} \mathbf{r}$. The CDFT generalization of the Levy-Lieb functional is the Vignale-Rasolt functional $F_{\mathrm{VR}}\left(\rho, \mathbf{j}_{\mathrm{p}}\right)$; there is likewise a generalization of the density-matrix constrained-search functional $F_{\mathrm{DM}}$ and the Lieb functional $F$ to include a current dependence. The mathematical properties of these functionals are not as well understood as in the standard DFT setting. The results presented in this article are in part meant to be a step towards such an understanding.

The article is structured as follows. In Sec.III, we describe DFT from a generic and abstract point of view, introducing arbitrary admissible density functionals $F_{0}$ and some concepts of convex analysis. We obtain several results that characterize the relationship between minimizers of the Rayleigh-Ritz variation principle and of the Hohenberg-Kohn variation principle. In Sec.III we apply our findings to the standard DFT of atoms and molecules in the Born-Oppenheimer approximation. The importance of the topology of the underlying density space is emphasized. In Sec.IV we discuss CDFT, and finally, in Sec.V we summarize and draw some conclusions.

\section{DFT FROM AN ABSTRACT POINT OF VIEW}

\section{A. Admissible universal density functionals}

Except for the trivial case of one electron, an explicit formula for any of the standard density functionals used in the Hohenberg-Kohn variation principle in Eq. (2) is not known. Moreover, the mathematical analysis of Eq. (2) is difficult without further assumptions. In his work [4], Lieb placed DFT for electronic systems in $\mathbb{R}^{3}$ on a firm mathematical ground using the language of convex analysis [9, 10], the natural setting for problems such as Eq. (22). The starting point is to embed the densities in a Banach space $X$ and to consider potentials in the dual space $X^{\prime}$, thereby making the interaction $(v \mid \rho)$ well-defined and continuous in both arguments. We then obtain the ground-state energy as a map $E: X^{\prime} \rightarrow \mathbb{R} \cup\{-\infty\}$ given by

$$
E(v)=\inf _{\rho \in X}\left(F_{0}(\rho)+(v \mid \rho)\right), \quad \forall v \in X^{\prime},
$$

where $F_{0}: X \rightarrow \mathbb{R} \cup\{+\infty\}$ is the universal density functional. Being the pointwise infimum of a collection of affine maps of the form $v \mapsto(v \mid \rho)+F_{0}(\rho)$, the ground-state energy in Eq. (7) is automatically concave and upper semi-continuous with respect to the topology on $X^{\prime}$. Note that we allow $E$ and $F_{0}$ to be infinite. The effective domain of a function is the set where the function is finite-for example, $\operatorname{dom}(E)=\left\{v \in X^{\prime} \mid\right.$ $E(v)>-\infty\}$.

In Ref. [4], Lieb (and Simon) proved several important results in the context where $X=L^{1}\left(\mathbb{R}^{3}\right) \cap L^{3}\left(\mathbb{R}^{3}\right)$, with dual $X^{\prime}=L^{3 / 2}\left(\mathbb{R}^{3}\right)+L^{\infty}\left(\mathbb{R}^{3}\right)$. However, this choice of $X$ is not unique, and the derived results may depend on $X$. We emphasize that, even if our notation suggests the standard DFT setting, it covers also CDFT and other settings.

A function $F_{0}: X \rightarrow \mathbb{R} \cup\{+\infty\}$ is said to be an admissible density functional if, for each potential $v \in X^{\prime}$, it gives a correct ground-state energy by the Hohenberg-Kohn variation principle in Eq. (7) - that is, if it produces identical results with the Rayleigh-Ritz variation principle in Eq. (10). Note that we do not require that there exists a minimizing density $\rho$ for a given potential $v$, even in those cases where $v$ supports a ground state in the Rayleigh-Ritz variation principle in Eq. (1). It is sufficient that a minimizing sequence $\left\{\rho_{n}\right\}$ can be found. Clearly, for any pair $(\rho, v) \in X \times X^{\prime}$, an admissible density functional and the ground-state energy satisfy the Fenchel inequality

$$
E(v) \leq F_{0}(\rho)+(v \mid \rho)
$$

We are here interested in characterizing those pairs $(\rho, v)$ that saturate Fenchel's inequality, $E(v)=F_{0}(\rho)+$ $(v \mid \rho)$; in other words, those $\rho \in X$ that are minimizers in Eq. (7) for a given $v \in X^{\prime}$.

Since the universal functional is in general not differentiable (see Refs. [4, 11] for the standard DFT case), we cannot write down an Euler equation for the solution of the minimization problem Eq. (7). Moreover, even if $F_{0}$ were differentiable, the Euler equation would in general be a necessary but not sufficient condition for a global minimum in Eq. (77). Instead, we use the concept of subdifferentiation to characterize the solution. 
The admissible density functional $F_{0}$ is said to be subdifferentiable at $\rho \in X$ if $F_{0}(\rho) \in \mathbb{R}$ and if there exists $u \in X^{\prime}$, known as a subgradient of $F_{0}$ at $\rho$, such that

$$
F_{0}\left(\rho^{\prime}\right) \geq F_{0}(\rho)+\left(u \mid \rho^{\prime}-\rho\right), \quad \forall \rho^{\prime} \in X,
$$

meaning that $F_{0}$ touches the affine map $\rho^{\prime} \mapsto F_{0}\left(\rho^{\prime}\right)+\left(u \mid \rho^{\prime}-\rho\right)$ at $\rho$ and lies nowhere below it. The subgradient is thus a generalization of the concept of the slope of a tangent to the graph of $F_{0}$ at $\rho$. The subdifferential $\partial^{-} F_{0}(\rho)$ is the (possibly empty) set of all subgradients of $F_{0}$ at $\rho$ :

$$
\partial^{-} F_{0}(\rho)=\left\{u \in X^{\prime} \mid F_{0}\left(\rho^{\prime}\right) \geq F_{0}(\rho)+\left(u \mid \rho^{\prime}-\rho\right), \forall \rho^{\prime} \in X, F_{0}(\rho) \in \mathbb{R}\right\} .
$$

This set is convex [9]. Note that the subdifferential is the empty set whenever $F_{0}(\rho)=+\infty$. Assuming that $-v$ is a subgradient of $F_{0}$ at $\rho$, we obtain by simple rearrangements

$$
\begin{aligned}
-v \in \partial^{-} F_{0}(\rho) & \Longleftrightarrow F_{0}\left(\rho^{\prime}\right) \geq F_{0}(\rho)+\left(-v \mid \rho^{\prime}-\rho\right) \\
& \Longleftrightarrow F_{0}(\rho)+(v \mid \rho) \leq F_{0}\left(\rho^{\prime}\right)+\left(v \mid \rho^{\prime}\right) \\
& \Longleftrightarrow F_{0}(\rho)+(v \mid \rho)=\inf _{\rho^{\prime} \in X}\left(F_{0}\left(\rho^{\prime}\right)+\left(v \mid \rho^{\prime}\right)\right)=E(v),
\end{aligned}
$$

We have thus shown the following sufficient and necessary condition for a minimizing density in the Hohenberg-Kohn variation principle in Eq. (7):

Proposition 1. Let $F_{0}: X \rightarrow \mathbb{R} \cup\{+\infty\}$ be an admissible density functional so that Eq. (7) holds. Let $v \in X^{\prime}$ and $\rho \in X$ be given. Then,

$$
E(v)=F_{0}(\rho)+(v \mid \rho) \Longleftrightarrow-v \in \partial^{-} F_{0}(\rho) .
$$

\section{B. Lieb's universal density functional}

From the Fenchel inequality in Eq. (8), we obtain by a trivial rearrangement the equivalent inequality

$$
F_{0}(\rho) \geq E(v)-(v \mid \rho),
$$

stating that each admissible density functional $F_{0}(\rho)$ is an upper bound to $\rho \mapsto E(v)-(v \mid \rho)$ with respect to all variations in $v \in X^{\prime}$. We now define the Lieb universal density functional $F(\rho)$ as the least upper bound to $E(v)-(v \mid \rho)$ for all $v \in X^{\prime}$ :

$$
F(\rho)=\sup _{v \in X^{\prime}}(E(v)-(v \mid \rho)), \quad \forall \rho \in X,
$$

which in the following will be referred as the Lieb variation principle. The Lieb functional is clearly a lower bound to all admissible density functionals:

$$
F(\rho) \leq F_{0}(\rho)
$$

Since the Lieb functional by construction also satisfies the Fenchel inequality in Eq. (8), we obtain:

Proposition 2. The Lieb functional is an admissible density functional:

$$
E(v)=\inf _{\rho \in X}(F(\rho)+(v \mid \rho)), \quad \forall v \in X^{\prime} .
$$

Proof. $E(v) \leq \inf _{\rho \in X}(F(\rho)+(v \mid \rho)) \leq \inf _{\rho \in X}\left(F_{0}(\rho)+(v \mid \rho)\right)=E(v)$.

The Lieb functional is related to the ground-state energy in a special, symmetrical manner-compare the Lieb variation principle in Eq. (14) with the Hohenberg-Kohn variation principle Eq. (16). In the language of convex analysis, $E$ and $F$ are said to be skew conjugate functions [12]: the function $E$ is concave and upper semi-continuous, whereas its skew conjugate function $F$ is convex and lower semi-continuous. Convexity of 
$F$ and concavity of $E$ mean that, for each pair $\rho_{1}, \rho_{2} \in X$, each pair $v_{1}, v_{2} \in X^{\prime}$, and each $\lambda \in(0,1)$, we have

$$
\begin{aligned}
& F\left(\lambda \rho_{1}+(1-\lambda) \rho_{2}\right) \leq \lambda F\left(\rho_{1}\right)+(1-\lambda) F\left(\rho_{2}\right), \\
& E\left(\lambda v_{1}+(1-\lambda) v_{2}\right) \geq \lambda E\left(v_{1}\right)+(1-\lambda) E\left(v_{2}\right),
\end{aligned}
$$

whereas lower semi-continuity of $F$ and upper semi-continuity of $E$ imply that

$$
\begin{gathered}
\liminf _{\rho \rightarrow \rho_{0}} F(\rho) \geq F\left(\rho_{0}\right), \\
\limsup _{v \rightarrow v_{0}} E(v) \leq E\left(v_{0}\right) .
\end{gathered}
$$

These properties follow straightforwardly from Eqs. (14) and (16). It is a fundamental result of convex analysis that there is a one-to-one correspondence between all lower semi-continuous convex functions on $X$ and all upper semi-continuous concave functions on $X^{\prime}$ [9, 10, 12], the correspondence being as in Eqs. (14) and (16). It follows that the Lieb functional $F$ is not only a lower bound to all admissible density functionals but also the only admissible density functional that is lower semi-continuous and convex with respect to the topology on $X$. It is a trivial but important observation that each property and each feature of $E$ are, in some manner, exactly reflected in the properties and features of $F$ and vice versa. Note, however, that the Lieb functional depends explicitly on $X$, just like $E$ depends on $X^{\prime}$.

Just like there may happen to be no minimizing density in the Hohenberg-Kohn variation principle, there may happen to be no maximizing potential in the Lieb variation principle. To characterize maximizing potentials, we introduce superdifferentiability by analogy with subdifferentiability. The ground-state energy $E$ is said to be superdifferentiable at $v \in X^{\prime}$ if there exists an element $\rho \in X$, known as a supergradient of $E$ at $v$, such that

$$
E\left(v^{\prime}\right) \leq E(v)+\left(v^{\prime}-v \mid \rho\right), \quad \forall v^{\prime} \in X^{\prime} .
$$

The superdifferential $\partial^{+} E(v)$ is the (possibly empty) convex set of all supergradients of $E$ at $v$ :

$$
\partial^{+} E(v)=\left\{\rho \in X \mid E\left(v^{\prime}\right) \leq E(v)+\left(\rho \mid v^{\prime}-v\right), \forall v^{\prime} \in X^{\prime}, E(v) \in \mathbb{R}\right\} .
$$

In exactly the same manner that we proved Eq. (12), we obtain the following necessary and sufficient conditions for the existence of a maximizing potential in the Lieb variation principle:

$$
E(v)=F(\rho)+(v \mid \rho) \Longleftrightarrow \rho \in \partial^{+} E(v) .
$$

Note carefully that this result holds only for the Lieb functional, not for an arbitrary admissible density functional. Combining Eqs. (12) and (23), we arrive at the following characterization of the optimality condition in Eqs. (14) and (16):

Proposition 3. If $F: X \rightarrow \mathbb{R} \cup\{+\infty\}$ is the Lieb functional and $E: X^{\prime} \rightarrow \mathbb{R} \cup\{-\infty\}$ is the ground-state energy, then

$$
E(v)=F(\rho)+(v \mid \rho) \Longleftrightarrow-v \in \partial^{-} F(\rho) \Longleftrightarrow \rho \in \partial^{+} E(v) .
$$

For a general admissible density functional $F_{0}$, these conditions are not equivalent: there may exist $\rho \in \partial^{+} E(v)$ such that $-v \notin \partial^{-} F_{0}(\rho)$ when $F_{0} \neq F$. On the other hand, the converse statement, $-v \in$ $\partial^{-} F_{0}(\rho) \Longrightarrow \rho \in \partial^{+} E(v)$, holds for any admissible density functional $F_{0}$. To prove this, assume that $-v \in \partial^{-} F_{0}(\rho)$. According to Eq. (12), we then have $F_{0}(\rho)=E(v)-(v \mid \rho)$. At the same time, the Fenchel inequality holds for any admissible density functional $F_{0}$ :

$$
E(u) \leq F_{0}(\rho)+(u \mid \rho), \quad \forall u \in X^{\prime} .
$$

Substituting $F_{0}(\rho)=E(v)-(v \mid \rho)$ in this inequality, we obtain

$$
E(u) \leq E(v)+(u-v \mid \rho), \quad \forall u \in X^{\prime},
$$

implying that $\rho \in \partial^{+} E(v)$. We have thus proved the following: 
Proposition 4. If $F_{0}: X \rightarrow \mathbb{R} \cup\{+\infty\}$ is an admissible functional and $E: X^{\prime} \rightarrow \mathbb{R} \cup\{-\infty\}$ the ground-state energy, then

$$
E(v)=F_{0}(\rho)+(v \mid \rho) \Longleftrightarrow-v \in \partial^{-} F_{0}(\rho) \Longrightarrow \rho \in \partial^{+} E(v) .
$$

In general, we have $F \leq F_{0}$. However, according to the following proposition, an admissible density functional $F_{0}(\rho)$ can differ from $F(\rho)$ only when $\partial^{-} F_{0}(\rho)=\emptyset$ :

Proposition 5. If $F_{0}: X \rightarrow \mathbb{R} \cup\{+\infty\}$ is an admissible functional and $F: X \rightarrow \mathbb{R} \cup\{+\infty\}$ the Lieb functional, then

$$
-v \in \partial^{-} F_{0}(\rho) \Longleftrightarrow F_{0}(\rho)=F(\rho) \wedge-v \in \partial^{-} F(\rho) .
$$

Proof. Let us assume that $-v \in \partial^{-} F_{0}(\rho)$. From Eq. (27), we then have $E(v)=F_{0}(\rho)+(v \mid \rho)$. Substituting this result into Eq. (24), we then find that $F(\rho)=F_{0}(\rho)$ and $-v \in \partial^{-} F(\rho)$. Conversely, if $F(\rho)=F_{0}(\rho)$ and $-v \in \partial^{-} F(\rho)$ hold, then we have $E(v)=F_{0}(\rho)+(v \mid \rho)$ by Eq. (24), from which $-v \in \partial^{-} F_{0}(\rho)$ follows by Eq. (27).

\section{Constrained-search density functionals}

So far, we have not established a connection between the minimizing densities in the Hohenberg-Kohn variation principle in Eq. (2) and the minimizing wave functions in the Rayleigh-Ritz variation principle in Eq. (11). For instance, we have not shown that each $\rho \in \partial^{+} E(v)$ is the ground-state density associated with some wave function $\psi$. Introducing a generic (abstract) constrained-search density functional, we consider in this section the relation between minimizers in the Hohenberg-Kohn and Rayleigh-Ritz variation principles.

Let $S$ be a set with elements $s$ called states and let $d: S \rightarrow X$ be a map from $S$ to the Banach space of densities $X$. The image

$$
\rho_{s}=d(s)
$$

is called the density of $s$. However, no physical meaning is attached to $s \in S$ or to $\rho \in X$ at this point; they are mathematical objects - for example, $\rho$ may be a (current) density or a reduced density matrix, while $s$ may be a pure state or a density operator. As before, $X^{\prime}$ is the dual space of $X$, meaning that $v \in X^{\prime}$ if and only if $v: X \rightarrow \mathbb{R}$ is a linear and continuous operator. Thus, the pairing $(v \mid \rho)$ is separately linear and continuous in $v$ and $\rho$. We call $v$ a potential but again no physical meaning is attached at present.

Assume next that, for every $v \in X^{\prime}$, we are given a map $\mathcal{E}_{v}: S \rightarrow \mathbb{R}$ of the form

$$
\mathcal{E}_{v}(s)=\mathcal{E}_{0}(s)+\left(v \mid \rho_{s}\right),
$$

where $\mathcal{E}_{0}: S \rightarrow \mathbb{R}$ is bounded below, meaning that there exists an $M \in \mathbb{R}$ such that

$$
\mathcal{E}_{0}(s) \geq M, \quad \forall s \in S
$$

We call $\mathcal{E}_{v}(s)$ the expectation value of the total energy when the system is in the state $s$ and influenced by the potential $v$. This setting covers all standard formulations of DFT and CDFT, including both pure-state and density-matrix formulations - in particular, we do not assume linearity of $\mathcal{E}_{0}(s)$ and $d(s)$ in $s$.

Next, define the ground-state energy $E: X^{\prime} \rightarrow \mathbb{R} \cup\{-\infty\}$ by the Rayleigh-Ritz variation principle:

$$
E(v):=\inf _{s \in S} \mathcal{E}_{v}(s)=\inf _{s \in S}\left(\mathcal{E}_{0}(s)+\left(v \mid \rho_{s}\right)\right) .
$$

Observing that $v$ couples to $\rho_{s}$ only during minimization, it makes sense to define the constrained-search functional $F_{\mathrm{CS}}: X \rightarrow \mathbb{R} \cup\{+\infty\}$ as the map

$$
F_{\mathrm{CS}}(\rho)=\inf \left\{\mathcal{E}_{0}(s) \mid s \mapsto \rho\right\},
$$


which takes the value $+\infty$ whenever there is no $s \in S$ with $\rho_{s}=\rho$. Combining Eqs. (32) and (33), we obtain the Hohenberg-Kohn variation principle

$$
E(v)=\inf _{\rho \in X}\left(F_{\mathrm{CS}}(\rho)+(v \mid \rho)\right) .
$$

This result proves:

Proposition 6. The constrained-search functional $F_{\mathrm{CS}}$ in Eq. (33) is an admissible density functional for the energy $E$ in $E q$. (32).

\section{Characterization of ground states}

Suppose that $v \in X^{\prime}$ is such that there exists $s \in S$ for which the infimum in Eq. (32) is a minimum:

$$
E(v)=\inf _{s^{\prime}} \mathcal{E}_{v}\left(s^{\prime}\right)=\mathcal{E}_{v}(s),
$$

meaning that there exists a ground state $s \in \arg \min _{s^{\prime}} \mathcal{E}_{v}\left(s^{\prime}\right)$ for $v$. Let now $v^{\prime} \in X^{\prime}$ be arbitrary, and compute

$$
E\left(v^{\prime}\right)=\inf _{s^{\prime} \in S} \mathcal{E}_{v^{\prime}}\left(s^{\prime}\right) \leq \mathcal{E}_{v^{\prime}}(s)=\mathcal{E}_{0}(s)+\left(v^{\prime} \mid \rho_{s}\right)=\mathcal{E}_{v}(s)+\left(v^{\prime}-v \mid \rho_{s}\right)=E(v)+\left(v^{\prime}-v \mid \rho_{s}\right),
$$

where we have used Eqs. (30) and (35). We have thus proved the following proposition:

Proposition 7. If $s \in S$ is a ground state for $v$ in Eq. (30), then $\rho_{s}$ is a supergradient of $E$ in Eq. (32) at $v$ :

$$
s \in \underset{s^{\prime}}{\arg \min } \mathcal{E}_{v}\left(s^{\prime}\right) \Longrightarrow \rho_{s} \in \partial^{+} E(v) .
$$

The following proposition establishes an important relationship between ground states and subgradients of $F_{\mathrm{CS}}$ :

Proposition 8. Let $F_{\mathrm{CS}}: X \rightarrow \mathbb{R} \cup\{+\infty\}$ be the constrained-search functional (33). If $s$ is the ground state for some potential $v \in X^{\prime}$ with density $\rho_{s}$, then $-v \in \partial^{-} F_{\mathrm{CS}}\left(\rho_{s}\right)$.

Proof. Let $s^{\prime} \in S$ with $\rho_{s^{\prime}}=\rho_{s}$. According to Eq. (32), we then have

$$
E(v)=\mathcal{E}_{0}(s)+\left(v \mid \rho_{s}\right) \leq \mathcal{E}_{0}\left(s^{\prime}\right)+\left(v \mid \rho_{s}\right) .
$$

Subtracting $\left(v \mid \rho_{s}\right)$ from both sides, taking the infimum on the right-hand side, and using the definition in Eq. (33), we obtain

$$
\mathcal{E}_{0}(s) \leq \inf _{s^{\prime} \mapsto \rho_{s}} \mathcal{E}_{0}\left(s^{\prime}\right)=F_{\mathrm{CS}}\left(\rho_{s}\right) \leq \mathcal{E}_{0}(s) .
$$

Thus, if there is a ground state $s \in S$ for $v \in X^{\prime}$, then

$$
F_{\mathrm{CS}}\left(\rho_{s}\right)+\left(v \mid \rho_{s}\right)=\mathcal{E}_{0}(s)+\left(v \mid \rho_{s}\right)=\mathcal{E}_{v}(s)=E(v) \leq F_{\mathrm{CS}}(\rho)+(v \mid \rho), \quad \forall \rho \in X .
$$

Rearranging, we obtain

$$
F_{\mathrm{CS}}(\rho) \geq F_{\mathrm{CS}}\left(\rho_{s}\right)-\left(v \mid \rho-\rho_{s}\right), \quad \forall \rho \in X,
$$

demonstrating that $-v \in \partial^{-} F_{\mathrm{CS}}\left(\rho_{s}\right)$ and completing the proof.

Conversely, does the subgradient relation $-v \in \partial^{-} F_{\mathrm{CS}}(\rho)$ imply that there exists a ground state $s \mapsto \rho$ ? To answer this question affirmatively, we must assume that $F_{\mathrm{CS}}$ is expectation valued, defined as follows: 
Definition 1 (Expectation-valued constrained-search functional). A constrained-search functional $F_{\mathrm{CS}}$ is called expectation valued if, for every $\rho$ with $F_{\mathrm{CS}}(\rho)<+\infty$, there exists an $s_{\rho} \in S$ such that

$$
F_{\mathrm{CS}}(\rho)=\inf _{s \mapsto \rho} \mathcal{E}_{0}(s)=\mathcal{E}_{0}\left(s_{\rho}\right), \quad s_{\rho} \mapsto \rho .
$$

In other words, if $F_{\mathrm{CS}}$ is expectation valued, then the infimum in Eq. (33) is a minimum if $F_{\mathrm{CS}}(\rho)<+\infty$, implying that $F_{\mathrm{CS}}(\rho)$ is the expectation value $\mathcal{E}_{0}(s)$ of some state $s \mapsto \rho$. If $F_{\mathrm{CS}}$ is expectation valued, it follows immediately that

$$
-v \in \partial^{-} F_{\mathrm{CS}}(\rho) \Longrightarrow E(v)=F_{\mathrm{CS}}(\rho)+(v \mid \rho) \Longrightarrow E(v)=\mathcal{E}_{0}\left(s_{\rho}\right)+(v \mid \rho)=\mathcal{E}_{v}\left(s_{\rho}\right) \Longrightarrow s_{\rho} \in \underset{s^{\prime}}{\arg \min } \mathcal{E}_{v}\left(s^{\prime}\right)
$$

and therefore that there exists a ground state $s \mapsto \rho$ of $v$. Summarizing, we have proved the following:

Proposition 9. Suppose that $F_{\mathrm{CS}}: X \rightarrow \mathbb{R} \cup\{+\infty\}$ is an expectation-valued constrained-search functional. Then, for each $v \in X^{\prime}$, we have

$$
s_{\rho} \in \underset{s^{\prime}}{\arg \min } \mathcal{E}_{v}\left(s^{\prime}\right) \Longleftrightarrow-v \in \partial^{-} F_{\mathrm{CS}}(\rho) \Longrightarrow \rho \in \partial^{+} E(v) .
$$

Remark: Proposition 9 for an expectation-valued constrained-search functional should be compared with Proposition 4 for a general admissible density functional. Whereas, for a general admissible density functional $F_{\mathrm{CS}}$, the condition $E(v)=F_{\mathrm{CS}}(\rho)+(v \mid \rho)$ does not imply the existence of a ground state $s \mapsto \rho$, this implication does hold for for an expectation-valued constrained-search functional. Compare also with Proposition 3, where the admissible density functional is assumed to be convex and lower semi-continuous (i.e., the Lieb functional).

Proposition 10. Suppose that an expectation-valued constrained-search functional $F_{\mathrm{CS}}: X \rightarrow \mathbb{R} \cup\{+\infty\}$ is convex and lower semi-continuous so that $F_{\mathrm{CS}}=F$. Then, for each $v \in X^{\prime}$, we have

$$
s_{\rho} \in \underset{s^{\prime}}{\arg \min } \mathcal{E}_{v}\left(s^{\prime}\right) \Longleftrightarrow-v \in \partial^{-} F_{\mathrm{CS}}(\rho) \Longleftrightarrow \rho \in \partial^{+} E(v) .
$$

Proof. Combine Propositions 3 and 9

\section{E. Differentiability of $E(v)$}

We consider here conditions for differentiability of $E: X^{\prime} \rightarrow \mathbb{R} \cup\{-\infty\}$. From Eq. (34), it follows that $E$ is a concave and upper semi-continuous function. Upper semi-continuity is, however, a rather weak property. What is needed to make $E$ continuous? It is a fact that a concave (convex) map over a Banach space is continuous on the interior of its domain [9]. Hence, if we can show that $E(v)$ is finite for every $v \in X^{\prime}$, then the domain $\operatorname{dom}(E)=X^{\prime}$ and continuity follows. The trick to show finiteness of $E(v)$ in the context of standard DFT is to observe that $s \mapsto \mathcal{V}_{v}(s)=\left(v \mid \rho_{s}\right)$ is relatively bounded with $\mathcal{E}_{0}$-bound smaller than one, meaning that, for each $v \in X^{\prime}$, there exist $\epsilon \in(0,1)$ and $C_{\epsilon} \geq 0$ such that

$$
\left|\mathcal{V}_{v}(s)\right| \leq \epsilon \mathcal{E}_{0}(s)+C_{\epsilon}, \quad \forall x \in S .
$$

Assuming that $\mathcal{V}_{v}$ is relatively bounded with $\epsilon<1$ and with no assumption on $C_{\epsilon}$, we obtain:

$$
\mathcal{E}_{v}(s)=\mathcal{E}_{0}(s)+\mathcal{V}_{v}(s) \geq \mathcal{E}_{0}(s)-\epsilon \mathcal{E}_{0}(s)-C_{\epsilon}=(1-\epsilon) \mathcal{E}_{0}(s)-C_{\epsilon} .
$$

Taking the infimum over $s \in S$ and using the fact that $\mathcal{E}_{0}(s)$ is by definition below bounded, we find

$$
E(v) \geq(1-\epsilon) M-C_{\epsilon}>-\infty .
$$

Proposition 11. Let $\mathcal{E}_{0}: S \rightarrow \mathbb{R}, E: X^{\prime} \rightarrow \mathbb{R} \cup\{-\infty\}$, and $\mathcal{V}_{v}: S \rightarrow \mathbb{R}$ be as described above. If $\mathcal{V}_{v}$ is relatively bounded with respect to $\mathcal{E}_{0}$ with bound $\epsilon<1$ for each $v \in X^{\prime}$, then $E$ is continuous on $X^{\prime}$. 
Proof. $E$ is everywhere finite, so $\operatorname{dom}(E)=X^{\prime}$. Therefore $E$ is continuous at any point $v \in X^{\prime}$.

Having determined sufficient conditions for continuity of $E$, what about differentiability? By definition, $E$ is superdifferentiable at $v \in X^{\prime}$ if the superdifferential $\partial^{+} E(v)$ is non-empty. Clearly, if $E$ is differentiable at $v$, the superdifferential is a singleton, $\partial^{+} E(v)=\{\nabla E(v)\}$. The converse is in general almost true [9]:

Theorem 1. Let $X$ be a Banach space. A convex (concave) map $f: X \rightarrow \mathbb{R} \cup\{-\infty,+\infty\}$ is Gâteaux differentiable at $x \in X$ if and only if it is continuous at $x$ with a unique subgradient (supergradient).

Hence a unique sub- or supergradient does not by itself guarantee continuity and therefore not (Gâteaux) differentiability: A subdifferential may be a singleton even if the function is non-differentiable. This subtle point illustrates the limitations of finite-dimensional intuition and has been the source of misunderstanding in the DFT literature. For a discussion, see Ref. [11] and references therein.

We can finally establish a useful statement on the differentiability of $E: X^{\prime} \rightarrow \mathbb{R} \cup\{-\infty\}$ :

Proposition 12. Let $E: X^{\prime} \rightarrow \mathbb{R}$ be everywhere finite and given by the Rayleigh-Ritz principle in Eq. (32) and assume that $F_{\mathrm{CS}}$ in Eq. (33) is expectation valued and equal to the Lieb functional (i.e., convex and lower semi-continuous). Then $E$ is differentiable at $v$ if and only if all ground states $s \in \operatorname{argmin}_{s^{\prime} \in S} \mathcal{E}_{v}\left(s^{\prime}\right)$ supported by $v$ have the same ground-state density, $s \mapsto \rho \in X$. In particular, $E$ is differentiable at $v$ if there exists a unique ground state $s$ for $v$.

Proof. Being continuous, $E$ is differentiable at $v$ if and only if $\partial^{+} E(v)$ is a singleton. But by Proposition 10 $\partial^{+} E(v)$ is a singleton if and only if all ground states of $v$ have the same density, using the assumption that $F_{\mathrm{CS}}$ is the Lieb functional.

Remark: The message here is that given the Rayleigh-Ritz variation principle, it is not sufficient to know that a potential $v \in X^{\prime}$ has a unique ground-state density $\rho=\rho_{s}$ to prove differentiability of $E$ at $v$. To identify the unique supergradient $\rho \in \partial^{+} E(v)$ with a ground-state density, $F_{\mathrm{CS}}$ must be identical with the Lieb functional (convex and lower semi-continuous) and expectation valued. If the Lieb functional happens to be different from every possible constrained-search functional, differentiability may fail, even if $v$ has a unique ground-state density $\rho_{s}$. A counterexample is given in Sec.III where we consider the Hilbert space $X_{\mathrm{H}}=L^{2}\left(\mathbb{R}^{3}\right)$ rather than the usual Banach space $X_{\mathrm{L}}=L^{1}\left(\mathbb{R}^{3}\right) \cap L^{3}\left(\mathbb{R}^{3}\right)$. Unlike $E: X_{\mathrm{L}} \rightarrow \mathbb{R}$, the function $E: X_{\mathrm{H}} \rightarrow \mathbb{R}$ is not differentiable.

Starting from a Hohenberg-Kohn variation principle with an arbitrary admissible density functional, it seems hard to prove differentiability from the sole assumption that $v$ has a unique ground-state density $\rho_{s}$. Identifying the proper constrained-search functional, based on the Rayleigh-Ritz variation principle, seems the only way out of the problem.

\section{APPLICATION TO DFT FOR MOLECULAR SYSTEMS}

\section{A. Ground states and ground-state densities}

For $N$-electron systems, each normalized wave function has a density $\rho \in L^{1}\left(\mathbb{R}^{3}\right)$ with $\rho \geq 0$ almost everywhere and $\int \rho(\mathbf{r}) \mathrm{d} \mathbf{r}=N$. The set of states $S$ can be taken to be either the $L^{2}$ normalized states $\psi \in$ $H_{\mathrm{S}}^{1}\left(\mathbb{R}^{3 N}\right)$ (pure states) or the set of density matrices $\Gamma=\sum_{k} \lambda_{k}\left|\psi_{k}\right\rangle\left\langle\psi_{k}\right|$ constructed as convex combinations from an orthonormal set $\left\{\psi_{k}\right\} \subset H_{\mathrm{S}}^{1}\left(\mathbb{R}^{3 N}\right)$ with $\lambda_{k} \geq 0$ and $\sum_{k} \lambda_{k}=1$ (mixed states). In the pure-state and mixed-state cases, respectively, constrained search gives the functionals $F_{\mathrm{LL}}$ defined in Eq. (3) and $F_{\mathrm{DM}}$ defined in Eq. (4):

$$
\begin{array}{rll}
F_{\mathrm{LL}}(\rho)=\inf _{\Psi \mapsto \rho} \mathcal{E}_{0}(\psi), & \mathcal{E}_{0}(\psi)=\langle\psi|\hat{T}+\hat{W}| \psi\rangle, & \mathcal{V}_{v}(\psi)=\langle\psi|\hat{V}| \psi\rangle=\left(\rho_{\psi} \mid v\right), \\
F_{\mathrm{DM}}(\rho)=\inf _{\Gamma \mapsto \rho} \mathcal{E}_{0}(\Gamma), & \mathcal{E}_{0}(\Gamma)=\operatorname{Tr}[(\hat{T}+\hat{W}) \Gamma], & \mathcal{V}_{v}(\Gamma)=\operatorname{Tr}(\Gamma \hat{V})=\left(\rho_{\Gamma} \mid v\right),
\end{array}
$$

where $\hat{V}$ is the multiplication operator associated with $v$. It is obvious that $F_{\mathrm{DM}} \leq F_{\mathrm{LL}}$. It is less obvious, but true, that there are $\rho \in L^{1}$ for which $F_{\mathrm{DM}}(\rho)<F_{\mathrm{LL}}(\rho)$. 
In a classic paper [13], Kato demonstrated that, for each $v \in L^{2}\left(\mathbb{R}^{3}\right)+L^{\infty}\left(\mathbb{R}^{3}\right)$, the $N$-electron Hamiltonian $\hat{H}=\hat{T}+\hat{W}+\hat{V}$ is self-adjoint on $L^{2}\left(\mathbb{R}^{3 N}\right)$ with domain $H_{\mathrm{S}}^{2}\left(\mathbb{R}^{3 N}\right)$ and bounded below. More generally, Simon demonstrated that, for each $v \in L^{3 / 2}\left(\mathbb{R}^{3}\right)+L^{\infty}\left(\mathbb{R}^{3}\right)$ (which includes all Coulomb potentials), $\mathcal{V}_{v}$ is relatively $\mathcal{E}_{0}$-bounded with an arbitrarily small bound [14]:

Theorem 2. Let $v \in L^{3 / 2}\left(\mathbb{R}^{3}\right)+L^{\infty}\left(\mathbb{R}^{3}\right)$ and let $\hat{V}$ be the corresponding multiplication operator. Then, for any $\epsilon>0$, there exists a constant $C_{\epsilon} \geq 0$, such that for all $\psi \in H_{\mathrm{S}}^{1}\left(\mathbb{R}^{3 N}\right)$ with $\|\psi\|_{2}=1$,

$$
|\langle\psi|\hat{V}| \psi\rangle| \leq \epsilon\langle\psi|\hat{T}+\hat{W}| \psi\rangle+C_{\epsilon} .
$$

Proof. See Ref. [14].

In the pure-state case, therefore, each $v \in L^{3 / 2}\left(\mathbb{R}^{3}\right)+L^{\infty}\left(\mathbb{R}^{3}\right)$ gives a $\mathcal{V}_{v}$ relatively bounded by $\mathcal{E}_{0}$, with bound $\epsilon$ arbitrarily small. Lieb proved that $F_{\mathrm{LL}}$ and $F_{\mathrm{DM}}$ are both expectation valued [4]. In the same publication, a proof (due to Simon) that $F_{\mathrm{DM}}: L^{1}\left(\mathbb{R}^{3}\right) \rightarrow \mathbb{R} \cup\{+\infty\}$ is convex and lower semi-continuous was given. Using the Sobolev embedding theorem, it can be shown that for each $\psi \in H_{\mathrm{S}}^{1}\left(\mathbb{R}^{3 N}\right)$, the corresponding density $\rho \leftarrow \psi$ belongs to $L^{3}\left(\mathbb{R}^{3}\right)[4]$. Thus, it is appropriate to consider the Banach space

$$
X_{\mathrm{L}}:=L^{1}\left(\mathbb{R}^{3}\right) \cap L^{3}\left(\mathbb{R}^{3}\right)
$$

with norm $\|\cdot\|=\|\cdot\|_{L^{1}}+\|\cdot\|_{L^{3}}$, whose dual space is

$$
X_{\mathrm{L}}^{\prime}=L^{3 / 2}\left(\mathbb{R}^{3}\right)+L^{\infty}\left(\mathbb{R}^{3}\right)
$$

with the topology induced by $\|\cdot\|[15]$. Since convergence in $X_{\mathrm{L}}$ implies convergence in $L^{1}, F_{\mathrm{DM}}: X_{\mathrm{L}} \rightarrow$ $\mathbb{R} \cup\{+\infty\}$ is lower semi-continuous as well. Applying Proposition 10 for $F_{\mathrm{DM}}$ (which is expectation valued and lower semi-continuous convex) and Proposition 9 for $F_{\mathrm{LL}}$ (which is expectation valued but not lower semi-continuous convex), we obtain

Corollary 1. Let $v \in X_{\mathrm{L}}^{\prime}=L^{3 / 2}\left(\mathbb{R}^{3}\right)+L^{\infty}\left(\mathbb{R}^{3}\right)$. Then,

$$
\begin{aligned}
\Gamma \in \underset{\Gamma^{\prime}}{\arg \min }\left(\mathcal{E}_{0}\left(\Gamma^{\prime}\right)+\left(\rho_{\Gamma^{\prime}} \mid v\right)\right) & \Longleftrightarrow-v \in \partial^{-} F_{\mathrm{DM}}\left(\rho_{\Gamma}\right) \Longleftrightarrow \rho_{\Gamma} \in \partial^{+} E(v), \\
\psi \in \underset{\psi^{\prime}}{\arg \min }\left(\mathcal{E}_{0}\left(\psi^{\prime}\right)+\left(\rho_{\psi^{\prime}} \mid v\right)\right) & \Longleftrightarrow-v \in \partial^{-} F_{\mathrm{LL}}\left(\rho_{\psi}\right) \Longrightarrow \rho_{\psi} \in \partial^{+} E(v) .
\end{aligned}
$$

Note that $\rho \in \partial^{+} E(v)$ does not imply the existence of a ground-state wave function for $v$ such that $\psi \mapsto \rho$, only the existence of a mixed ground state $\Gamma \mapsto \rho$. For example, if $v \in X_{\mathrm{L}}^{\prime}$ has a two-fold degeneracy with pure ground states $\psi_{1}$ and $\psi_{2}$, then $\lambda \rho_{1}+(1-\lambda) \rho_{2} \in \partial^{+} E(v)$ but there may be no pure ground state with this density. On the other hand, $\lambda\left|\psi_{1}\right\rangle\left\langle\psi_{1}|+(1-\lambda)| \psi_{2}\right\rangle\left\langle\psi_{2}\right|$ is a mixed ground state with this density.

\section{B. Topology dependence of the Lieb functional}

Recall that the Lieb functional $F: X \rightarrow \mathbb{R} \cup\{+\infty\}$ depends explicitly on the Banach space $X$, including its topology. We now demonstrate this using an explicit example. Motivated by the set inclusion

$$
X_{\mathrm{L}}=L^{1}\left(\mathbb{R}^{3}\right) \cap L^{3}\left(\mathbb{R}^{3}\right) \subset X_{\mathrm{H}}:=L^{2}\left(\mathbb{R}^{3}\right)
$$

and the fact that $X_{\mathrm{H}}$ is Hilbert space with a simpler structure than the non-reflexive space $X_{\mathrm{L}}$, it is tempting to consider $F_{\mathrm{DM}}$ as a function on $X=X_{\mathrm{H}}$. However, the embedding $X_{\mathrm{L}} \subset X_{\mathrm{H}}$ is not continuous: Convergence in $X_{\mathrm{L}}$ does not imply convergence in $X_{\mathrm{H}}$. Even if $F_{\mathrm{DM}}$ is lower semi-continuous with respect to $X_{\mathrm{L}}$, it may fail to be so in $X_{\mathrm{H}}$. To see this, we note that, since $X_{\mathrm{H}}$ is a Hilbert space, $X_{\mathrm{H}}^{\prime}=X_{\mathrm{H}}$, which does not admit constant potentials: If $v \in X_{\mathrm{H}}$, then $v+c \notin X_{\mathrm{H}}$ for each constant $c \neq 0$. This observation allows us to prove the following result: 
Proposition 13. For each $v \in X_{\mathrm{H}}$, we have $E(v) \leq 0$. If $v \geq 0$ almost everywhere, then $E(v)=0$.

Proof. See Appendix A.

The proof is based on the following idea. Write $v=v_{+}-v_{-}$where $v_{ \pm} \geq 0$ almost everywhere. The negative part $v_{-}$can only lower the energy, whereas $v_{+} \in X_{\mathrm{H}}$ implies that $v$ decays at infinity (in an average sense), thereby allowing the electrons to lower their energy to zero by "escaping to infinity". Note how the fact that nonzero $c \notin X_{\mathrm{H}}$ affects this argument.

Consider now $\rho \equiv 0$, for which $F_{\mathrm{DM}}(\rho)=+\infty$ since no $\Gamma \mapsto 0$. Evaluating the conjugate (Lieb) functional with respect to $X_{\mathrm{H}}$ at $\rho=0$, we obtain

$$
F(0)=\sup _{v \in X_{\mathrm{H}}}(E(v)-(v \mid 0))=\sup _{v \in X_{\mathrm{H}}} E(v)=0 .
$$

Note that a different result is obtained using $X_{\mathrm{L}}$, which admits constant potentials $v(\mathbf{r}) \equiv c \in \mathbb{R}$ :

$$
F(0)=\sup _{v \in X_{\mathrm{L}}}(E(v)-(v \mid 0))=\sup _{v \in X_{\mathrm{L}}} E(v)=+\infty=F_{\mathrm{DM}}(0) .
$$

As an immediate consequence, we arrive at the following result on $X_{\mathrm{H}}$ :

Proposition 14. For any $v \in X_{\mathrm{H}}$ such that $v \geq 0$ almost everywhere, it holds that,

$$
E(v)=F(0)+(v \mid 0) \wedge-v \in \partial^{-} F(0) \wedge 0 \in \partial^{+} E(v),
$$

where $F$ is conjugate to $E$. There exists no ground state $\psi \in H_{\mathrm{S}}^{1}\left(\mathbb{R}^{3 N}\right)$ for any $v \geq 0$.

Proof. Eq. (59) follows from Proposition[3] It remains to show that there exists no ground state if $v \geq 0$ almost everywhere. Since $E(v)=0$, it is sufficient to show that, for each $\psi \in H_{\mathrm{S}}^{1}\left(\mathbb{R}^{3 N}\right)$, it holds that $\mathcal{E}_{v}(\psi)>0$. We have $\mathcal{E}_{v}(\psi) \geq\langle\psi|\hat{T}| \psi\rangle$. But if $\psi$ has zero kinetic energy, then $\nabla \psi=0$, from which it follows that $\psi=0$ almost everywhere, contradicting $\|\psi\|_{2}=1$. Thus $\mathcal{E}_{v}(\psi) \geq\langle\psi|\hat{T}| \psi\rangle>0$.

Our discussion illustrates how the choice of Banach space $X$ of densities affects the properties of the conjugate universal functional $F: X \rightarrow \mathbb{R} \cup\{+\infty\}$. Clearly, the Lieb functional $F_{\mathrm{DM}}$ for the space $X_{\mathrm{L}}$ is very different from the Lieb functional for $X_{\mathrm{H}}$, since, in the latter case, for any non-negative potential $v$, the unphysical density $\rho \equiv 0$ is a minimizer of the Hohenberg-Kohn variation principle. But $v$ does not even have a ground state and certainly not a ground state with density $\rho \equiv 0$.

Connecting with the discussion following Proposition 12 we can see how topology influences differentiability of $E$. The map $E: X_{\mathrm{H}} \rightarrow \mathbb{R}$ is pointwise identical to $E: X_{\mathrm{L}}^{\prime} \rightarrow \mathbb{R}$ : it is by definition the ground-state energy of the system in the external potential $v$, defined via the Rayleigh-Ritz variation principle. However, the topology on $X_{\mathrm{H}}$ is different and $F_{\mathrm{DM}}$ is no longer equal to the Lieb functional. We then cannot conclude from Proposition 12 that $E: X_{\mathrm{H}} \rightarrow \mathbb{R}$ is differentiable at $v$ if $v \in X_{\mathrm{H}}$ has a unique ground state.

\section{APPLICATION TO CDFT FOR MOLECULAR SYSTEMS IN MAGNETIC FIELDS}

Consider an $N$-electron system subject to an external magnetic vector potential $\mathbf{A}: \mathbb{R}^{3} \rightarrow \mathbb{R}^{3}$, with associated magnetic field $\mathbf{B}(\mathbf{r})=\nabla \times \mathbf{A}(\mathbf{r})$ (in the distributional sense). Considering $\mathbf{A}$ as a variable on the same footing as the scalar potential $v$, we arrive at CDFT, where the paramagnetic current density $\mathbf{j}_{\mathrm{p}} \in \mathbf{L}^{1}\left(\mathbb{R}^{3}\right)$ appears as a variable together with $\rho[6,68,16]$. The mathematical foundation of CDFT is not as well developed as that of DFT. Indeed, part of the motivation for the present work stems from a study of CDFT [17]. On the other hand, Laestadius [8] has taken important steps-proving, for example, Proposition 17 below.

Following Ref. [8], we assume for simplicity that the components of $\mathbf{A}$ are $L^{\infty}\left(\mathbb{R}^{3}\right)$ functions. The singleelectron momentum operator $-\mathrm{i} \nabla$ is then replaced by $-\mathrm{i} \nabla+\mathbf{A}$, transforming the $N$-electron kinetic-energy operator $\hat{T}$ into the corresponding $\hat{T}_{\mathbf{A}}$. Since $\mathbf{A} \in \mathbf{L}^{\infty}\left(\mathbb{R}^{3}\right)$, the kinetic-energy expectation value is still well 
defined for each $\psi \in H_{\mathrm{S}}^{1}\left(\mathbb{R}^{3 N}\right)$. In terms of the density $\rho \in L^{1}\left(\mathbb{R}^{3}\right)$ and the paramagnetic current density $\mathbf{j}_{\mathrm{p}} \in \mathbf{L}^{1}\left(\mathbb{R}^{3}\right)$, we obtain

$$
\left\langle\psi\left|\hat{T}_{\mathbf{A}}\right| \psi\right\rangle=\langle\psi|\hat{T}| \psi\rangle+\frac{1}{2} \int|\mathbf{A}(\mathbf{r})|^{2} \rho(\mathbf{r}) \mathrm{d} \mathbf{r}+\int \mathbf{A}(\mathbf{r}) \cdot \mathbf{j}_{\mathbf{p}}(\mathbf{r}) \mathrm{d} \mathbf{r} .
$$

The ground-state energy is thus given by

$$
E(v, \mathbf{A})=\inf _{\psi,\|\psi\|_{2}=1}\left\langle\psi\left|\hat{T}_{\mathbf{A}}+\hat{W}+\hat{V}\right| \psi\right\rangle=\inf _{\left(\rho, \mathbf{j}_{\mathrm{p}}\right)}\left(F_{\mathrm{VR}}\left(\rho, \mathbf{j}_{\mathrm{p}}\right)+\left(v+\frac{1}{2} A^{2} \mid \rho\right)+\left(\mathbf{A} \mid \mathbf{j}_{\mathrm{p}}\right)\right),
$$

where we have introduced the Vignale-Rasolt (VR) functional [6]

$$
F_{\mathrm{VR}}\left(\rho, \mathbf{j}_{\mathrm{p}}\right):=\inf \left\{\langle\psi|\hat{T}+\hat{W}| \psi\rangle \mid \psi \in H_{\mathrm{S}}^{1}\left(\mathbb{R}^{3 N}\right),\|\psi\|_{2}=1, \psi \mapsto\left(\rho, \mathbf{j}_{\mathrm{p}}\right)\right\}
$$

Similarly, we may define a density-matrix constrained-search functional

$$
F_{\mathrm{DM}}\left(\rho, \mathbf{j}_{\mathrm{p}}\right)=\inf \left\{\operatorname{Tr}((\hat{T}+\hat{W}) \Gamma) \mid \Gamma \mapsto\left(\rho, \mathbf{j}_{\mathrm{p}}\right)\right\},
$$

where $\rho_{\Gamma}=\sum_{k} p_{k} \rho_{\psi_{k}}$ and $\mathbf{j}_{\mathrm{p}_{\Gamma}}=\sum_{k} p_{k} \mathbf{j}_{\mathrm{p}_{\psi_{k}}}$. Whereas $F_{\mathrm{DM}}$ is convex by construction, the presence of the nonlinear A-dependent term makes $E(v, \mathbf{A})$ nonconcave. Following Ref. [16], it is therefore natural instead to work with $\tilde{E}(u, A)=E\left(u-\frac{1}{2} A^{2}, \mathbf{A}\right)$, defined in Proposition [16 below. We note that

$$
\left(\rho, \mathbf{j}_{\mathrm{p}}\right) \in X_{\mathrm{L}} \times \mathbf{L}^{1}\left(\mathbb{R}^{3}\right),
$$

a Banach space with norm $\left\|\left(\rho, \mathbf{j}_{\mathrm{p}}\right)\right\|=\|\rho\|+\left\|\mathbf{j}_{\mathrm{p}}\right\|_{\mathbf{L}^{1}}$ and dual $X_{\mathrm{L}}^{\prime} \times \mathbf{L}^{\infty}\left(\mathbb{R}^{3}\right)$, the latter containing all $(v, \mathbf{A})$. First, we prove finiteness of the ground-state energy:

Proposition 15. For every $(v, \mathbf{A}) \in X_{\mathrm{L}} \times \mathbf{L}^{\infty}$, the ground-state energy is finite, $E(v, \mathbf{A})>-\infty$.

Proof. For an $N$-electron system influenced by a magnetic field, the diamagnetic inequality [18] gives

$$
\langle\phi|\hat{T}| \phi\rangle \leq\left\langle\psi\left|\hat{T}_{\mathbf{A}}\right| \psi\right\rangle, \quad \forall \psi \in H_{\mathrm{S}}^{1}\left(\mathbb{R}^{3 N}\right),
$$

where $\phi=|\psi|$ be the pointwise absolute value. Next, using the identity $\rho_{\psi}=\rho_{\phi}$ and the relative boundedness of $v \in X_{\mathrm{L}}$ with respect to $\hat{T}$, we find that $\hat{V}$ is relatively bounded with respect to $\hat{T}_{\mathbf{A}}$ :

$$
|\langle\psi|\hat{V}| \psi\rangle|=|\langle\phi|\hat{V}| \phi\rangle| \leq \epsilon\langle\phi|\hat{T}| \phi\rangle+C_{\epsilon} \leq \epsilon\left\langle\psi\left|\hat{T}_{\mathbf{A}}\right| \psi\right\rangle+C_{\epsilon} .
$$

Similarly, $\hat{W}$ is relatively bounded with respect to $\hat{T}_{\mathbf{A}}$. It follows that $\langle\psi|\hat{H}| \psi\rangle$ is below bounded, and thus that $E(v, \mathbf{A})>-\infty$.

Next, we note that, for each $\mathbf{A} \in \mathbf{L}^{\infty}\left(\mathbb{R}^{3}\right)$, we have $|\mathbf{A}|^{2} \in L^{\infty}\left(\mathbb{R}^{3}\right) \subset X_{\mathrm{L}}^{\prime}$. Therefore, for each pair $(v, \mathbf{A}) \in X_{\mathrm{L}}$, we have $\left(v \pm \frac{1}{2}|\mathbf{A}|^{2}, \mathbf{A}\right) \in X_{\mathrm{L}} \times \mathbf{L}^{\infty}$, making the following proposition easy to prove:

Proposition 16. If $F_{0}: X_{L} \times \mathbf{L}^{1}$ is either $F_{\mathrm{VR}}$ or $F_{\mathrm{DM}}$, then $\tilde{E}: X_{\mathrm{L}}^{\prime} \times \mathbf{L}^{\infty}\left(\mathbb{R}^{3}\right) \rightarrow \mathbb{R}$ defined by

$$
\tilde{E}(u, \mathbf{A})=\inf _{\left(\rho, \mathbf{j}_{p}\right)}\left(F_{0}\left(\rho, \mathbf{j}_{p}\right)+(u \mid \rho)+\left(\mathbf{A} \mid \mathbf{j}_{p}\right)\right)
$$

is concave, finite, and therefore continuous. The ground-state energy $E$ and $\tilde{E}$ are related by

$$
E(v, \mathbf{A})=\tilde{E}\left(v+\frac{1}{2}|\mathbf{A}|^{2}, \mathbf{A}\right), \quad \tilde{E}(u, \mathbf{A})=E\left(u-\frac{1}{2}|\mathbf{A}|^{2}, \mathbf{A}\right) .
$$

Proof. We leave the details to the reader. 
The map $(v, \mathbf{A}) \mapsto\left(v+\frac{1}{2}|\mathbf{A}|^{2}, \mathbf{A}\right)$ defined on $X_{\mathrm{L}}^{\prime} \times \mathbf{L}^{\infty}\left(\mathbb{R}^{3}\right)$ is smooth and invertible, with smooth inverse $(u, \mathbf{A}) \mapsto\left(u-\frac{1}{2}|\mathbf{A}|^{2}, \mathbf{A}\right)$. Thus, the properties of $E$ are reflected in properties $\tilde{E}$ and vice versa. If $F_{0}$ is an admissible functional for $\tilde{E}: X_{\mathrm{L}}^{\prime} \times \mathbf{L}^{\infty} \rightarrow \mathbb{R}$, then

$$
\begin{aligned}
E(v, \mathbf{A})=F_{0}\left(\rho, \mathbf{j}_{\mathrm{p}}\right)+\left(v+\frac{1}{2}|\mathbf{A}|^{2} \mid \rho\right)+\left(\mathbf{A} \mid \mathbf{j}_{\mathrm{p}}\right) & \Longleftrightarrow \quad-\left(v+\frac{1}{2}|\mathbf{A}|^{2}, \mathbf{A}\right) \in \partial^{-} F_{0}\left(\rho, \mathbf{j}_{\mathrm{p}}\right) \\
& \Longrightarrow \quad\left(\rho, \mathbf{j}_{\mathrm{p}}\right) \in \partial^{+} \tilde{E}\left(v+\frac{1}{2}|\mathbf{A}|^{2}, \mathbf{A}\right)
\end{aligned}
$$

Laestadius proved the following result $[8]$ :

Proposition 17. $F_{\mathrm{VR}}: L^{1}\left(\mathbb{R}^{3}\right) \times \mathbf{L}^{1}\left(\mathbb{R}^{3}\right) \rightarrow \mathbb{R} \cup\{+\infty\}$ is expectation valued.

Proposition 9 therefore gives

$$
\psi_{\rho} \in \underset{\psi^{\prime}}{\arg \min }\langle\psi|\hat{H}| \psi\rangle \quad \Longleftrightarrow \quad E(v, \mathbf{A})=F_{\mathrm{VR}}\left(\rho, \mathbf{j}_{\mathrm{p}}\right)+\left(v+\frac{1}{2}|\mathbf{A}|^{2} \mid \rho\right)+\left(\mathbf{A} \mid \mathbf{j}_{\mathrm{p}}\right) .
$$

Thus, using the Vignale-Rasolt functional and the space $X_{\mathrm{L}} \times \mathbf{L}^{1}$ as density space, there are no minimizers of the pure-state Hohenberg-Kohn variation principle for CDFT that do not correspond to ground-state wave functions.

On the other hand, it is not known whether the density-matrix functional $F_{\mathrm{DM}}$ in (62) is expectation valued, but it seems likely. If $F_{\mathrm{DM}}$ is not expectation valued, there may be $\left(\rho, \mathbf{j}_{\mathrm{p}}\right)$ such that

$$
E(v, \mathbf{A})=F_{\mathrm{DM}}\left(\rho, \mathbf{j}_{\mathrm{p}}\right)+\left(v+\frac{1}{2}|\mathbf{A}|^{2} \mid \rho\right)+\left(\mathbf{A} \mid \mathbf{j}_{\mathrm{p}}\right)
$$

but such that there is no $\Gamma \mapsto\left(\rho, \mathbf{j}_{\mathrm{p}}\right)$, i.e., $\left(\rho, \mathbf{j}_{\mathrm{p}}\right)$ must be considered an unphysical minimizer of the densitymatrix Hohenberg-Kohn variation principle for CDFT.

Neither is it known whether $F_{\mathrm{DM}}$ is lower semi-continuous in the $L^{1} \times \mathbf{L}^{1}$ topology; the proof for the ordinary DFT case in Ref. [4] is not easy to generalize to the present situation. Thus, there could be $\left(\rho, \mathbf{j}_{\mathrm{p}}\right) \in \partial^{+} \tilde{E}\left(v+\frac{1}{2}|\mathbf{A}|^{2}, \mathbf{A}\right)$ such that $-\left(v+\frac{1}{2}|\mathbf{A}|^{2}, \mathbf{A}\right) \notin \partial^{-} F_{\mathrm{DM}}\left(\rho, \mathbf{j}_{\mathrm{p}}\right)$, so that $\left(\rho, \mathbf{j}_{\mathrm{p}}\right)$ does not satisfy Eq. (71).

Finally, we may consider the Lieb functional $F: X_{\mathrm{L}} \times \mathbf{L}^{1} \rightarrow \mathbb{R} \cup\{+\infty\}$, defined as

$$
F\left(\rho, \mathbf{j}_{\mathrm{p}}\right):=\sup _{u, \mathbf{A}}\left(\tilde{E}(u, \mathbf{A})-(u \mid \rho)-\left(\mathbf{A} \mid \mathbf{j}_{\mathrm{p}}\right)\right)=\sup _{v, \mathbf{A}}\left(E(v, \mathbf{A})-\left(v+\frac{1}{2}|\mathbf{A}|^{2} \mid \rho\right)-\left(\mathbf{A} \mid \mathbf{j}_{\mathrm{p}}\right)\right) .
$$

This functional is convex and lower semi-continuous by construction. However, unlike in standard DFT, it is unknown whether $F\left(\rho, \mathbf{j}_{\mathrm{p}}\right)=F_{\mathrm{DM}}\left(\rho, \mathbf{j}_{\mathrm{p}}\right)$.

From the perspective of applying Proposition 10, thereby establishing a result like Corollary 1 for CDFT, one must establish both that $F_{\mathrm{DM}}$ is expectation valued and lower semi-continuous. Thus, we do not know at the present time, whether $E(v, \mathbf{A})$ is Gâteaux-differentiable when the ground-state density $\left(\rho, \mathbf{j}_{\mathrm{p}}\right)$ is unique.

\section{CONCLUSION}

We have analyzed the relationship between ground-state densities obtained from the Rayleigh-Ritz variation principle (by minimizing over pure-state wave functions or mixed-state density matrices) and from the Hohenberg-Kohn variation principles (by minimizing over densities using an admissible density functional $\left.F_{0}(\rho)\right)$. For standard DFT for molecular systems, we established, for each potential $v \in L^{3 / 2}\left(\mathbb{R}^{3}\right)+L^{\infty}\left(\mathbb{R}^{3}\right)$, a one-to-one correspondence between the mixed ground-state densities of the Rayleigh-Ritz variation principle and the mixed ground-state densities of the Hohenberg-Kohn variation principle with the Lieb densitymatrix constrained-search functional $F_{\mathrm{DM}}: L^{1}\left(\mathbb{R}^{3}\right) \cap L^{3}\left(\mathbb{R}^{3}\right) \rightarrow \mathbb{R} \cup\{+\infty\}$. A similar one-to-one correspondence is established between the pure ground-state densities of the Rayleigh-Ritz variation principle and the pure ground-state densities of the Hohenberg-Kohn variation principle with the Levy-Lieb functional $F_{\mathrm{LL}}: L^{1}\left(\mathbb{R}^{3}\right) \cap L^{3}\left(\mathbb{R}^{3}\right) \rightarrow \mathbb{R}$. In other words, all physical ground-state densities (pure or mixed) are recovered with these functionals and there are no false densities (i.e., minimizing densities not associated with a ground-state wave function). 
We also noted how the topology of the underlying Banach space $X$ impinges on the results - in particular, we noted that the Lieb functional $F: X \rightarrow \mathbb{R} \cup\{+\infty\}$ depends explicitly on $X$. As an illustration, $F \neq F_{\mathrm{DM}}$ on $X=L^{2}\left(\mathbb{R}^{3}\right)$ but $F=F_{\mathrm{DM}}$ on $L^{1}\left(\mathbb{R}^{3}\right) \cap L^{3}\left(\mathbb{R}^{3}\right)$. Finally, CDFT was discussed and some open problems were pointed out - for example, it is unknown whether $F_{\mathrm{DM}}\left(\rho, \mathbf{j}_{\mathrm{p}}\right)$ is lower semi-continuous in any useful topology such as $L^{1}\left(\mathbb{R}^{3}\right) \times \mathbf{L}^{1}\left(\mathbb{R}^{3}\right)$.

\section{ACKNOWLEDGMENTS}

This work was supported by the Norwegian Research Council through the CoE Centre for Theoretical and Computational Chemistry (CTCC) Grant No. 179568/V30 and the Grant No. 171185/V30 and through the European Research Council under the European Union Seventh Framework Program through the Advanced Grant ABACUS, ERC Grant Agreement No. 267683.

\section{Appendix A: Proof of Proposition 13}

Proof of Proposition 13. Writing $v=v_{+}-v_{-}$, we obtain

$$
E(v)=\inf _{\rho}\left(F_{\mathrm{DM}}(\rho)+\left(v_{+} \mid, \rho\right)-\left(v_{-} \mid, \rho\right)\right) \leq \inf _{\rho}\left(F_{\mathrm{DM}}(\rho)+\left(v_{+} \mid \rho\right)=E\left(v_{+}\right)\right) .
$$

It is therefore sufficient to show that $E\left(v_{+}\right) \leq 0$. In fact, we show that $E\left(v_{+}\right)=0$.

Let $v \geq 0$ almost everywhere. Let $\lambda>0$ be arbitrary and let $\Omega_{k, \lambda}$ with $k \in \mathbb{N}$ be disjoint cubes of side length $\lambda$ such that $\cup_{k} \Omega_{k, \lambda}=\mathbb{R}^{3}$. Each $\Omega_{k, \lambda}$ can be obtained by translation of $\Omega_{1, \lambda}$. Since $v \in L^{2}\left(\mathbb{R}^{3}\right)$,

$$
\int_{\mathbb{R}^{3}} v(\mathbf{r})^{2} \mathrm{~d} \mathbf{r}=\sum_{k} \int_{\Omega_{k, \lambda}} v(\mathbf{r})^{2} \mathrm{~d} \mathbf{r}<+\infty,
$$

implying that $\int_{\Omega_{k, \lambda}} v(\mathbf{r})^{2} \mathrm{~d} \mathbf{r} \rightarrow 0$ as $k \rightarrow \infty$. Let $\psi \in \mathcal{C}_{c}^{\infty}\left(\mathbb{R}^{3 N}\right)$ be arbitrary but with support in $\Omega_{1,1}^{N}$ so that $\rho_{\psi} \in \mathcal{C}_{c}^{\infty}\left(\mathbb{R}^{3}\right)$ has support contained in $\Omega_{1,1}$. By translating $\psi$ properly (denoting the result by $\psi_{k}$ ), the support of $\psi_{k}$ is inside $\Omega_{k, 1}^{N}$ and

$$
F_{\mathrm{DM}}\left(\rho_{\psi_{k}}\right)=F_{\mathrm{DM}}\left(\rho_{\psi}\right) \leq\langle\psi|T+W| \psi\rangle \equiv\langle T\rangle+\langle W\rangle,
$$

independent of $k$. We obtain

$$
E(v) \leq \lim _{k}\left(\langle T\rangle+\langle W\rangle+\int_{\Omega_{k, 1}} v(\mathbf{r}) \rho_{k}(\mathbf{r}) \mathrm{d} \mathbf{r}\right)=\langle T\rangle+\langle W\rangle
$$

where we have used the fact that

$$
\int_{\Omega_{k, 1}} v(\mathbf{r}) \rho_{k}(\mathbf{r}) \mathrm{d} \mathbf{r} \leq\left(\int_{\Omega_{k, 1}} v(\mathbf{r})^{2} \mathrm{~d} \mathbf{r}\right)^{1 / 2}\|\rho\|_{2} \rightarrow 0
$$

as $k \rightarrow \infty$.

We now increase the size of the boxes $\Omega_{k, \lambda}$ by varying $\lambda>0$. By dilating $\psi$ in the manner

$$
\psi\left(\mathbf{r}_{1}, \cdots\right) \mapsto \lambda^{3 N / 2} \psi\left(\lambda \mathbf{r}_{1}, \cdots\right),
$$

the support is still inside $\Omega_{1, \lambda}^{\infty}$ and the density is scaled as $\rho_{\psi}(\mathbf{r}) \rightarrow \lambda^{-3} \rho_{\psi}\left(\lambda^{-1} \mathbf{r}\right)$, conserving the number of particles. We obtain the scaling

$$
\langle T\rangle+\langle W\rangle \rightarrow \lambda^{-2}\langle T\rangle+\lambda^{-1}\langle W\rangle .
$$

By repeating the above argument for $\lambda=1$ and letting $\lambda \rightarrow \infty$, we obtain $E(v) \leq 0$. On the other hand, $E(v) \geq 0$ since the Hamiltonian $H(v)$ is positive with $v \geq 0$, yielding $E(v)=0$. 
[1] L. Thomas, Proc. Camb. Philos. Soc. 23, 542 (1927).

[2] E. Fermi, Rend. Accad. Naz. Lincei 6, 602 (1927).

[3] P. Hohenberg and W. Kohn, Phys. Rev. 136, B864 (1964).

[4] E. H. Lieb, Int. J. Quant. Chem. 24, 243 (1983).

[5] M. Levy, Proc. Natl. Acad. Sci. 76, 6062 (1979).

[6] G. Vignale and M. Rasolt, Phys. Rev. Lett. 59, 2360 (1987).

[7] G. Vignale and M. Rasolt, Phys. Rev. B 37, 10685 (1988).

[8] A. Laestadius, Int. J. Quantum Chem. 114, 1445 (2014).

[9] J. van Tiel, Convex Analysis, an Introductory Text (Wiley, Chichester, 1984).

[10] I. Ekeland and R. Témam, Convex Analysis and Variational Problems (SIAM, Philadelphia, 1999).

[11] P. Lammert, Int. J. Quant. Chem. 107, 1944 (2005).

[12] R. Rockafellar, Pac. J. Math. 25, 597 (1968).

[13] T. Kato, Trans. Amer. Math. Soc. 70, 195 (1951).

[14] B. Simon, Commun. Math. Phys. 21, 192 (1971).

[15] T.-S. Liu and J.-K. Wang, Math. Scand. 23, 241 (1968).

[16] E. Tellgren, S. Kvaal, E. Sagvolden, U. Ekstrm, A. Teale, and T. Helgaker, Phys. Rev. A 86, 062506 (2012)

[17] S. Kvaal and T. Helgaker, (2015), in preparation.

[18] E. Lieb and M. Loss, Analysis, 2nd ed. (American Mathematical Society, Providence, Rhode Island, USA, 2001). 